\title{
Why do skeletons lie about their age?
}

\author{
Ian Magee
}

Department of Archaeology, UCC

\section{Introduction}

Age is, essentially, a dichotomy of socially defined hierarchies and chronological measurement. On the one hand, an individual is labelled according to a socially defined passage of time (e.g., infant, adult, etc...), which reflects their level of participation within the community, and, on the other, according to a chronologically measured passage of time. Whilst these elements are mutually exclusive in their construct, to varying degrees they are dependent on each other for their definition. It is the variation in these definitions that presents a bioarchaeological paradox. The labels of one element have been borrowed by the other, resulting in semantic confusion of social and metric labelling, which has negatively impacted on any attempt to answer one of the most basic of osteological inquiries, age-at-death estimation.

Is a 14 year old in India, who is betrothed, works full time and contributes financially to the household an adult or child? Is a 30 year old in Ireland, who is single, unemployed and who lives in the parental home an adult or a child? Different cultures will define them very differently. In the forensic analysis of human skeletal remains, there is little room for such subjectivity. As such, the intransigence of metric definition disenfranchises the individual of much of their identity and much of what defines the culture of which they were a part. Attempting to identify the activities in which an individual was engaged over their lifetime will go some way to redressing that imbalance.

\section{Hypothesis}

Fundamental to any forensic investigation of archaeologically retrieved human skeletal remains is the estimation of age-at-death. Because of known chronologies for bone growth and tooth eruption, pre-adult and young adult age estimation is a fairly straightforward and accurate procedure. On the other hand, methodologies used to establish an accurate age-at-death of adult skeletal remains apply chronological age to the natural maturation and degenerative processes of specific anatomical points of reference. However, these methodologies have been shown individually to be inaccurate through consistently overaging younger adults and under-aging older adults (by up to 20 years). 
The primary aim of this research is to determine why methodologies which incorporate anatomical sites subject to general repetitive lifetime activities (as most of them are) give consistently inaccurate age-at-death estimations.

One of the anatomical points of reference used by osteoarchaeologists to estimate age-atdeath in adults is the auricular (ear-shaped) surface of the sacro-iliac joint. The joint is located where the sacrum (base of spine) joins the ilium (top part) of the os coxae (pelvic bones). Patterns and combinations of different features (for example, pores and bony growths) can be seen on individual joint surfaces. It is the presence or absence of these patterns and combinations that are associated with different age groups.

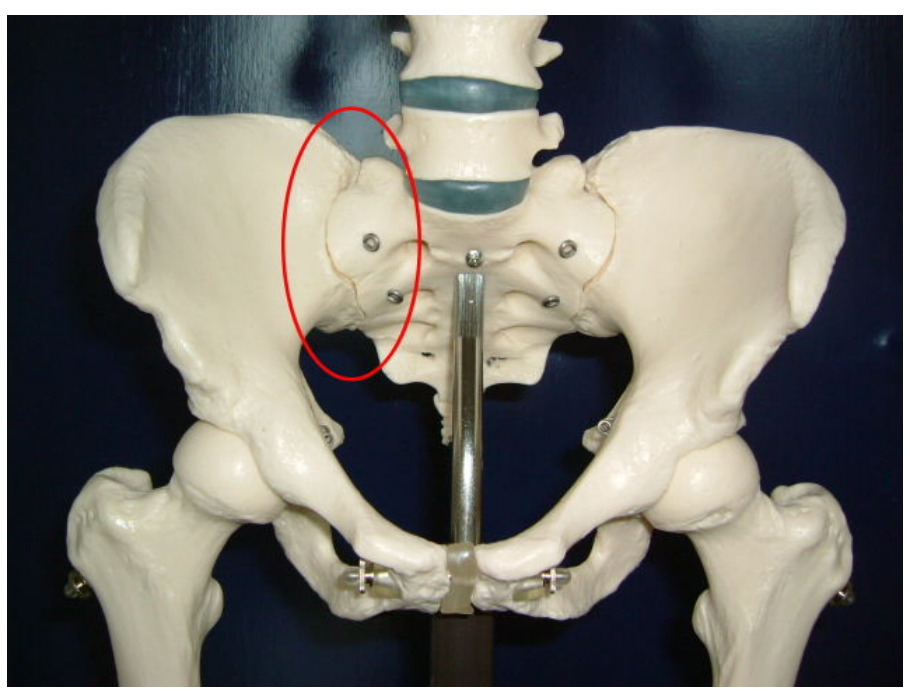

Figure 1: Sacro-Iliac Joint

This joint is particularly important to osteoarchaeologists because of its high rate of preservation in archaeological contexts. Because the joint is held in place by strong ligaments, the surfaces of the joint are protected during the decaying process and subsequent 'settling' of earth surrounding the individual.

However, methodologies motivated by this joint have shown to be as inaccurate as the other techniques available to forensic anthropology, and, as with techniques using other anatomical points of reference, the pattern of inaccuracy has so far not been satisfactorily explained.

Research carried out by radiologists has demonstrated that, far from being a completely fixed joint, as previously thought, there is a downwards movement during walking, while the hips rotate from side to side creating a shearing effect (Kampen and Tillman 1998). Given the function of the sacro-iliac joint in weight bearing and weight distribution to the lower limbs, it is argued that repetitive occupational stress from the individual human experience has a fundamental bearing on development and degeneration of the joint and in particular on features on the auricular surface, irrespective of chronological aging. 


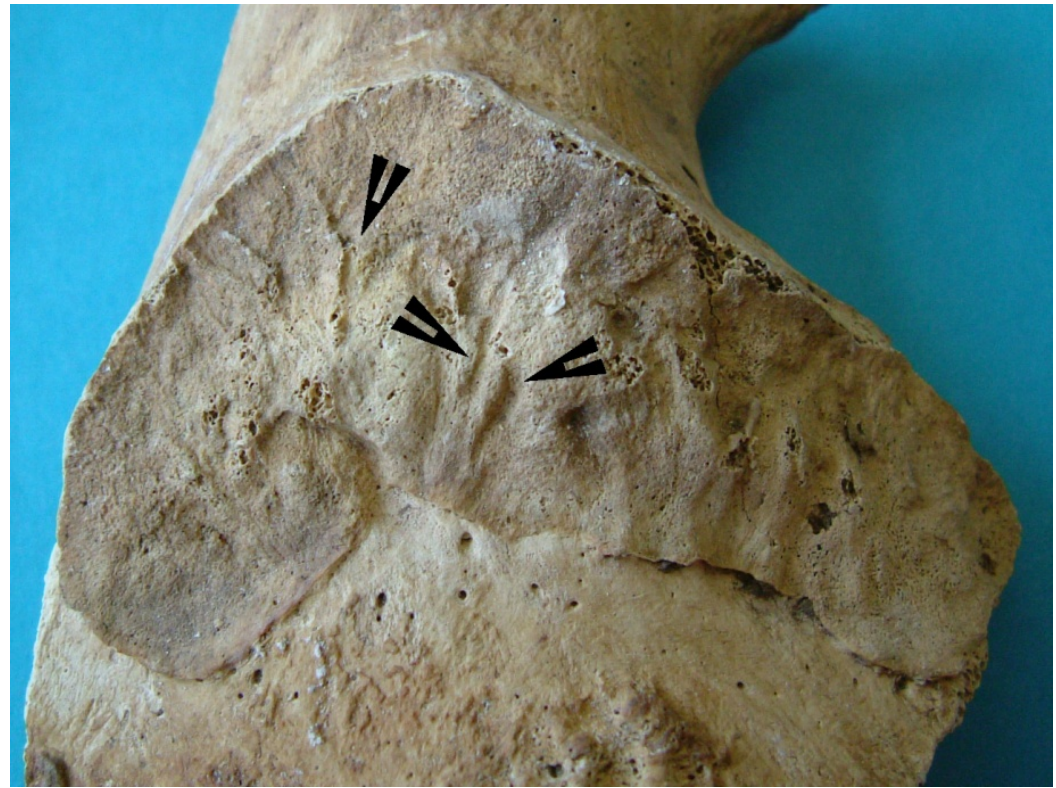

Figure 2: Auricular surface showing 'Groove' feature.

\section{Materials Used}

An assemblage of skeletal remains of known age at death was archaeologically retrieved from the crypt of Christ Church, Spitalfields, in London. The collection amounts to a total of 987 individuals. Of those, 250 are of known age-at-death, sex and occupation (unique in an archaeologically retrieved collection). These include a wide variety of occupations, including those involving heavy lifting and manual labour. This assemblage was added to a comparative sample from the University of Coimbra, Portugal, of individuals from the late 19th and early 20th centuries of known age-at-death and occupation.

Twenty-six features on and around each auricular surface were investigated and tested as to association with pre-defined occupation types. Many of these features were common to all previous methodologies. Several previously ignored features were included, as they showed the potential to be influential in the formation of the different patterns used for estimating age-at-death. These included markers on the bone surface that indicate where ligaments and muscles are attached. For these particular features, the premise is simple: the larger the marker, the stronger the attachment, the bigger the muscle, the more biomechanically loaded the activities that led to the muscle growth. The individual occupation types were grouped according to four basic groups, representing activities subject to decreasing definitions of assumed mechanical stress: Artisans, representing, for example, manual labourers; Domestics, representing light manual activities; Master Craftsmen/Weavers, representing skilled work that did not involve the heaviest of assumed mechanical loading; Professionals, the latter assuming a limited lifetime exposure to biomechanical stress. 


\section{Findings}

Of the 26 features that were investigated, all demonstrated different degrees of association with specific occupation groupings. Some features were significantly associated with a particular individual occupation type. It should be possible to statistically refine the specific occupations from within each group in order to isolate similar long term activity types from past populations.

Some features were associated with all occupation group types but to either decreasing or increasing degrees of significance, depending on the assumed biomechanical loads associated with each occupation group. These patterns were also demonstrated within broad age groups. This shows that the rate of appearance of certain features on the auricular feature is influenced less by actual aging than by the biomechanical stress involved in certain occupation types and the time span over which those activities are regularly performed.

\section{Conclusion}

The impact of repetitive occupational stress determines which features on and around joint surfaces are the result of habitual activities over an extended period of time during an individual's life. Where these features are used in methodologies to estimate age-at-death, a labelling bias is unavoidable when an attempt is made to assign a chronological age to an individual. Isolation of the bias will facilitate more accurate forensic interpretation of age of an individual and the potential to identify even broadly definable activity types within human skeletal remains which will benefit not just forensic and osteoarchaeological interpretation of the individual, but also demographic interpretation at a community and cultural level.

The theorisation of 'age' in archaeological thought has facilitated debate on the degree to which the application of a chronology to biological remains is influenced by cultural and social definition of age, and the activities associated with it. Within the debate, there is a resistance to the idea that features on bone surface derived from muscle and ligament attachments are clear indicators of specific cultural or occupational activities. Research has already indicated muscle stress markers for activities as specific as kayaking and horse riding and more recently on specific occupations such as weaving. One main point of contention in the debate, however, is the suggestion that collective activities will have a cumulative effect on the skeleton in respect of bone change.

This research has shown that not only does the stress from repetitive occupational activity leave specific features on the bone surface, but that they are in fact responsible for the inaccuracies in forensic methodologies for estimating age-at-death. As a consequence, it shows the need to address the evidence of lifetime activity on human skeletal remains in a 
manner which requires a reappraisal of our definition of the role of our ancestors in their community and how this can be best portrayed outside of the age labelling bias of the present.

I wish to acknowledge the help and support of Rob Kruszynski at the National History Museum, London, and all the staff in the Department of Anthropology at the University of Coimbra, especially Dr. Ana Luisa Santos. I am grateful, also, to Beverly Schmidt at the University Of Connecticut for her encouragement and patience and thank Dr. Barra Ó Donnabháin for his supervision. 